\title{
28 Research Soure \\ Recovery Sleep Attenuates Impairments in Working Memory Following Total Sleep Deprivation
}

\author{
Zi-Yi Peng \\ Beijing Sport University \\ Yi Wang \\ Beijing Sport University \\ Ci-Min Dai \\ Beijing Sport University \\ Le-Tong Wang \\ Beijing Sport University \\ Song-Yue Xie \\ Beijing Sport University \\ Jia-Lu Li \\ Beijing Sport University \\ Ling-Jing Zeng \\ Beijing Sport University \\ Yong-Cong Shao ( $\sim$ budeshao@hotmail.com ) \\ Beijing Sport University \\ Wei-Wei Fu \\ Chinese Academy of Sciences
}

\section{Research}

Keywords: sleep deprivation, recovery sleep, working memory, event-related potential, N2, P3

Posted Date: October 9th, 2020

DOI: https://doi.org/10.21203/rs.3.rs-88727/v1

License: (c) (i) This work is licensed under a Creative Commons Attribution 4.0 International License. Read Full License 


\section{Abstract}

Background: Considering the well-known detrimental effects of sleep deprivation (SD) on cognitive function and quality of life, sleep disturbances represent a major physical and mental health issue. Because working memory plays an important role in many complex cognitive processes, it is necessary to identify strategies that can effectively counteract the negative effects of SD on working memory.

Methods: In the present study, we utilized event-related potentials (ERPs) to investigate the restorative effect of $8 \mathrm{~h}$ of recovery sleep (RS) on working memory impairments induced by $36 \mathrm{~h}$ of total sleep deprivation (TSD). We analyzed data from 16 healthy male participants. All participants completed a 2-back working memory task before and after $36 \mathrm{~h}$ of TSD and after $8 \mathrm{~h}$ of RS. Electroencephalography (EEG) data were recorded during the task.

Results: N2 and P3 components related to working memory exhibited low-amplitude slow-wave characteristics following $36 \mathrm{~h}$ of TSD. Significant decreases in the latency of the N2 component were observed after $8 \mathrm{~h}$ of RS. RS also induced significant increases in the amplitude of the P3 component, along with significant decreases in P3 latency.

Conclusions: Eight hours of RS also attenuated decreases in working memory performance caused by $36 \mathrm{~h}$ of TSD. However, the effects of RS appear to be limited.

\section{Background}

Although proper sleep has been shown to exert beneficial effects on memory, cognitive function, work performance, and immune-related parameters $[1,2,3,4]$, the incidence of sleep problems continues to increase. Sleep deprivation (SD) or lack of sleep is a very common phenomenon in military activities. It is one of the important factors leading to fatigue, loss of combat effectiveness, and personal losses. While the most direct and obvious behavioral manifestation of SD is drowsiness, SD is also known to significantly impair cognitive function [5, 6, 7, 8]. Honn et al. further demonstrated that SD decreases processing speed in visual search, spatial memory, paired associative learning, motor response, and other types of cognitive tasks [9]. Additional evidence has indicated that SD significantly increase response times in working memory tasks and that such changes are accompanied by decreased activation of the frontoparietal cortex (FPC), which plays an important role in cognitive control. Specifically, the FPC can bypass top-down cognitive control, enabling individuals to focus on goal-related information while suppressing irrelevant information $[10,11]$.

Working memory is a limited-capacity system involved in the temporary storage and maintenance of information related to a specific task [12], acting as a bridge between short-term and long-term memory [13]. The working memory system also plays a role in operating, processing, and executing control. Previous studies have consistently demonstrated that SD significantly impairs working memory $[14,15]$. In addition to decreasing the quality of information stored in working memory, SD slows processing speed and alters event-related potentials (ERPs) during task performance by prolonging the latency and reducing the amplitude of the N2 and P3 components [16]. Such changes have also been associated with decreases in the ability to discriminate amongst target stimuli [17], reduced availability of disposable attentional resources, and alterations in selective attention toward emotional stimuli/signal processing [18].

Considering these findings, numerous research groups have aimed to identify interventions that can effectively counteract the negative consequences of SD. Although caffeine and other drugs can effectively maintain work 
performance and alertness [19,20,21], their effects on high-level cognitive functions are quite limited, and the use of caffeine may be associated with recovery costs in individuals with long-term sleep deficiency [22]. Recovery sleep (RS) refers to a short period of adequate sleep that follows SD, representing a potential non-pharmacological strategy for combating the effects of SD on cognitive function. Some studies have reported that RS can attenuate hyperalgesia [23 24] and cognitive impairment [25] due to SD. Such studies have also reported that RS alleviates fatigue and improves attention/alertness, and that longer periods of RS are associated with greater restoration of cognitive function [26]. Indeed, one study reported that $8 \mathrm{~h}$ of RS can attenuate impairments in response inhibition caused by $36 \mathrm{~h}$ of total sleep deprivation (TSD) [27]. One night of TSD leads to obvious changes in the topological characteristics of the small-world network in the brain. Although two nights of RS can completely restore the global properties of the brain network, such RS does not appear to induce changes in local function [28]. Impairments in sleep structure following $58 \mathrm{~h}$ of wakefulness have also been shown to improve following $14 \mathrm{~h}$ of RS [29]. A recent study reported that a 90-min nap during a day of sleep deprivation can restore hippocampus-dependent learning, and that the structural composition of the hippocampus predicts the success of learning recovery [30]. Other studies have suggested that one or two nights of RS after complete or chronic sleep loss cannot sufficiently attenuate neurobehavioral deficits, self-monitoring abilities, and decreases in brain metabolism [31, 32, 33]. Thus, the amount of sleep required to restore cognitive function following extended wakefulness remains unclear.

Impairments in cognitive function due to SD will inevitably affect task performance. Previous studies have relied on both neurophysiological and behavioral indicators to assess an individual's physiological and psychological state. Among them, neurophysiological indicators are more sensitive for assessing the effects of SD. Chai et al. (2020) reported that two nights of RS after one night of TSD restored hippocampal connectivity to normal levels, but did not fully restore behavioral performance or its associations with hippocampal connectivity [34]. In an investigation of joint rhythm, [35] reported that significant differences in RON (the reorienting negativity) amplitude relative to behavioral responses can be explained by the increased sensitivity of neurophysiological measures. N2 is considered to reflect performance monitoring and cognitive control (top-down and bottom-up), and its neural source has most consistently been identified as the anterior midcingulate cortex (aMCC) [36, 37]. Some authors have proposed that the P300 plays a crucial role in processing at the intersection between perception and decisionmaking [38], while others have suggested that it more commonly plays a role in synthesizing information and carrying information related to conscious access, attentional moderation, or post-response adaptations [39, 40, 41]. Additional studies ahve revealed that the P3 wave is caused by the renewal of working memory content and decreases with increases in working memory load [42].

In summary, it remains to be determined whether $8 \mathrm{~h}$ of RS can attenuate SD-induced impairments in working memory. In the present study, we aimed to investigate the potentially restorative effects of RS on working memory and ERP latency/amplitude following $36 \mathrm{~h}$ of TSD. We hypothesized that such $8 \mathrm{~h}$ of RS would partially attenuate the deleterious effects of $36 \mathrm{~h}$ of TSD on working memory in our participants: compared with $36 \mathrm{~h}$ of TSD, $8 \mathrm{~h}$ of RS will decrease the latencies of the working memory-related N2-P3 components and increase their amplitudes, but the differences between $36 \mathrm{~h}-\mathrm{TSD}$ and $8 \mathrm{~h}-\mathrm{RS}$ might not statistically significant, suggesting that the restorative effects of $8 \mathrm{~h}$ of RS are limited.

\section{Materials And Methods 2.1 Participants}


The present study included 16 healthy male college students (mean age: 23 years; age range: 21-28 years). Although the effects of TSD on N2/P3 components related to working memory were reported in a previous study [43], the RS data are presented here for the first time. All participants were right-handed and maintained good sleep habits (Pittsburgh Sleep Quality Index (PSQI) < 5 points) [44]. None reported a history of mental or physical illness, and none had previously undergone psychophysiological testing. All participants had normal or corrected-to-normal visual acuity, and their IQ scores were greater than the population average (Raven Test scores >110 points). Our research staff provided a full explanation of the study procedures prior to the experiment. All participants were asked to refrain from smoking, drinking alcohol/coffee, and taking any drugs for at least $48 \mathrm{~h}$ prior to the experiment, and to maintain a normal sleep pattern for 1 week. All participants reported sleeping for 7-9 h per day, between 10:00 pm and 9:00 am. Participants' sleep routines throughout the study were confirmed using sleep diaries. All participants provided written informed consent and received monetary compensation upon completion of the experiment. The study was approved by the ethics committee of the former Fourth Military Medical University.

\subsection{Experimental design}

The present study included a 2-back pronunciation working memory (Fig. 1A), a 2-back spatial working memory (Fig. 1B), and a 2-back object working memory (Fig. 1C) task. Stimuli for these tasks included 15 case-insensitive English letters (excluding similar letters), small black squares, and 12 geometric figures, respectively. Each task lasted approximately $5 \mathrm{~min}$ and included 122 trials. Target stimuli were presented for $400 \mathrm{~ms}$, with an inter-stimulus interval of 1,600 ms. Participants were asked to match the current stimulus with the stimulus presented two trials earlier. Participants were instructed to press the left mouse button in the case of consistent stimuli and the right mouse button in the case of inconsistent stimuli. Consistent and inconsistent stimuli were presented in pseudorandom order in a 1:1 ratio.

\subsection{Experimental procedures}

We adopted a pre-post design, which is widely utilized in sleep research. Participants underwent $36 \mathrm{~h}$ of TSD, following which they underwent $8 \mathrm{~h}$ of RS (Fig. 2). Prior to testing, all participants practiced the experimental tasks until reaching an accuracy $>90 \%$, in order to exclude the influence of practice. Participants slept in the laboratory the night prior to completing the experimental tasks, and all participants were instructed to sleep from 11:00 pm to 7:00 am to ensure a sleep time of $8 \mathrm{~h}$. TSD was initiated at 8:00 am the next morning. The 2-back working memory tasks were performed both before and after TSD, and after $8 \mathrm{~h}$ of RS. EEG data were recorded simultaneously. To avoid sleep inertia, RS occurred from 11:00 pm to 7:00 am, and the third set of EEG data was acquired at 8:00 am on the third day [45]. Previous research has demonstrated that individual performance is relatively impaired within 5 min of awakening, gradually returning to normal over a period of 15 to 30 minutes [46].Two participants completed the experiment at the same time. Two medical workers and one researcher were present throughout the TSD period to prevent the participant from sleeping/napping. During the experiment, participants were allowed to eat, drink, and perform light physical activities, but were not allowed to engage in strenuous exercise or to ingestion caffeine, alcohol, or tea.

\subsection{EEG recordings}

EEG data were acquired in a dark, sound-proof, and electronically shielded EEG laboratory using 64-electrode caps. Stimuli were generated and presented using Stim-2 software (NeuroScan). Electrodes were arranged in accordance 
with the international 10-20 system. Horizontal and vertical electrooculograms were also recorded during EEG acquisition, and the bilateral mastoid process was used as the reference electrode. Recordings were performed at $1,000 \mathrm{~Hz}$. Channel impedance was maintained below $5 \mathrm{k} \Omega$.

\subsection{Data analysis}

ERP data could not be recorded in two cases due to technical issues. Data from these two participants were excluded during post-processing.

EEG data were pre-processed using SCAN4.3 software, following which ocular artifacts were removed via regression analysis. The data were bandpass filtered at $0.05-30 \mathrm{~Hz}$, with a frequency slope of $24 \mathrm{~dB} /$ oct. The data were divided into 900 -ms epochs (-100 ms to $800 \mathrm{~ms}$ ). The period of $100 \mathrm{~ms}$ prior to stimulation was included for baseline correction. Trials in which the voltage exceeded $\pm 100 \mu \mathrm{V}$ were rejected. The mean number of accepted trials was $92.4 \pm 17.46$. The P3 (250-450 ms) and N2 components (150-350 ms) were analyzed for the following channels: F3, Fz, F4, C3, Cz, C4, P3, Pz, and P4.

Repeated-measures analyses of variance (ANOVA) were used to analyze both behavioral data and ERP findings through SPSS v.22. For ERP analyses, we assessed main effects and interactions for sleep states ( $36 \mathrm{~h}$ TSD and $8 \mathrm{~h}$ RS), tasks (pronunciation working memory, spatial working memory, and object working memory), regions (frontal, central, and parietal), and sites (left, middle, and right). We analyzed behavioral parameters including mean reaction time, accuracy, and number of correct responses per unit of time. Behavioral data were compared between the two sleep states (36 h TSD and $8 \mathrm{~h}$ RS) and among the three types of tasks (pronunciation working memory, spatial working memory, and object working memory). Greenhouse-Geisser corrections for non-sphericity and Bonferroni post hoc tests were performed. Results are presented as the mean and standard deviation.

\section{Results}

\subsection{Behavioral performance}

Mean reaction times, accuracy, and number of correct responses per unit time are presented in Table 1. Although mean reaction times tended to decrease after $8 \mathrm{~h}$ of RS when compared with those observed after $36 \mathrm{~h}$ of TSD, this difference was not statistically significant $\left(F_{(1,13)}=2.144, P=0.167, \eta^{2}{ }_{P}=0.412\right)$, However, we observed significant increases in accuracy $\left(F_{(1,13)}=10.617, P=0.006, \eta^{2} \mathrm{P}=0.450\right)$ and the number of correct responses per unit of time $\left(F_{(1 \otimes 13)}=6.260, P=0.026, \eta_{\mathrm{P}=0.325}^{2}\right)$ after $8 \mathrm{~h}$ of $\mathrm{RS}$. No other main effects or interaction effects reached statistical significance.

Table 1

Behavioral performance (mean \pm SD) on the 2-back task after $36 \mathrm{~h}$ of TSD and after $8 \mathrm{~h}$ of RS

\begin{tabular}{|llll|}
\hline & Oh & 36hTSD & 8hRS \\
\hline Mean Reaction time(ms) & $507.26(82.04)$ & $542.77(103.73)$ & $515.85(82.47)$ \\
\hline Correct rate(\%) & $0.94(0.04)$ & $0.85(0.12)$ & $0.92(0.04)$ \\
\hline Correct number/sec & $1.91(0.35)$ & $1.64(0.42)$ & $1.84(0.34)$ \\
\hline
\end{tabular}

\subsection{N2 component}


Descriptive statistics for the N2 component are presented in Tables 2 and 3. Compared with the results obtained following $36 \mathrm{~h}$ of TSD, $8 \mathrm{~h}$ of RS was associated with significant decreases in N2 latency $\left(F_{(1,13)}=7.170, P=0.019\right.$, $\left.\eta^{2}{ }_{\mathrm{P}}=0.355\right)$ (Fig. 3, Fig. 4B). Our analyses further revealed a significant main effect of region on $\mathrm{N} 2\left(F_{(2 \ltimes 26)}=10.689\right.$, $\left.P=0.002, \eta_{\mathrm{P}}^{2}=0.451\right)$, indicating that the $\mathrm{N} 2$ latency was longer in the frontal region than in the central $(P=0.003)$ or parietal regions $(P=0.007)$ (Fig. 3). Although $\mathrm{N} 2$ amplitude also increased after $8 \mathrm{~h}$ of $\mathrm{RS}\left(F_{(1 \otimes 13)}=0.337, P=\right.$ $\left.0.571, \eta^{2}=0.025\right)$, this difference was not statistically significant (Table 3, Fig. 4A). However, N2 amplitudes were more negative in left electrodes than in right electrodes $\left(F_{(2 \times 26)}=7.211, P=0.003, \eta^{2}{ }_{P}=0.357\right)$. No other main effects or interaction effects reached statistical significance.

Table 2

Grand-average peak latency of the N2 and P3 components for correct responses across multiple electrode sites after $36 \mathrm{~h}$ of TSD and after $8 \mathrm{~h}$ of RS

\begin{tabular}{|c|c|c|c|c|c|c|c|}
\hline & & \multicolumn{2}{|l|}{$\mathrm{Oh}$} & \multicolumn{2}{|l|}{36 h-TSD } & \multicolumn{2}{|l|}{8 h-RS } \\
\hline & & N2 & P3 & N2 & P3 & N2 & P3 \\
\hline \multirow[t]{2}{*}{ F3 } & $\mathrm{M}(\mathrm{SD})$ & $245.05(27.17)$ & $376.55(20.25)$ & $262.01(40.37)$ & $379.11(25.86)$ & 240.91 & 368.81 \\
\hline & & & & & & $(28.13)$ & $(29.22)$ \\
\hline \multirow[t]{2}{*}{$\mathrm{Fz}$} & $\mathrm{M}(\mathrm{SD})$ & $243.36(25.49)$ & $374.75(19.90)$ & $260.72(31.55)$ & $382.70(29.48)$ & 241.14 & 364.05 \\
\hline & & & & & & $(24.20)$ & $(28.18)$ \\
\hline \multirow[t]{2}{*}{$\mathrm{F} 4$} & $\mathrm{M}(\mathrm{SD})$ & $239.46(27.33)$ & $373.20(19.92)$ & 261.86(39.24) & $380.26(25.93)$ & 238.90 & 368.12 \\
\hline & & & & & & $(29.14)$ & $(27.07)$ \\
\hline \multirow[t]{2}{*}{ C3 } & $M(S D)$ & 234.64(28.98) & $370.71(19.19)$ & $238.39(40.16)$ & $363.80(32.50)$ & 226.78 & 354.81 \\
\hline & & & & & & $(29.32)$ & (37.10) \\
\hline \multirow[t]{2}{*}{$\mathrm{Cz}$} & $M(S D)$ & $236.33(31.09)$ & $362.17(24.53)$ & $238.04(40.83)$ & $366.19(42.65)$ & 233.73 & 346.74 \\
\hline & & & & & & $(27.56)$ & (31.79) \\
\hline \multirow[t]{2}{*}{$\mathrm{C} 4$} & $M(S D)$ & $230.75(33.37)$ & $368.18(18.65)$ & $242.18(44.43)$ & $365.59(35.13)$ & 232.81 & 350.11 \\
\hline & & & & & & (32.99) & $(29.99)$ \\
\hline \multirow[t]{2}{*}{ P3 } & $M(S D)$ & $218.71(54.68)$ & $354.11(29.25)$ & $213.39(41.47)$ & $357.09(44.99)$ & 219.64 & 343.29 \\
\hline & & & & & & $(45.45)$ & (35.34) \\
\hline \multirow[t]{2}{*}{$\mathrm{Pz}$} & $M(S D)$ & $213.57(37.03)$ & $350.28(34.14)$ & $229.17(46.15)$ & $341.13(41.06)$ & 214.67 & 334.14 \\
\hline & & & & & & (37.68) & (37.86) \\
\hline \multirow[t]{2}{*}{ P4 } & $M(S D)$ & $221.30(54.50)$ & $326.72(38.87)$ & $227.36(50.51)$ & $324.11(48.03)$ & 221.59 & 323.25 \\
\hline & & & & & & $(53.10)$ & (38.78) \\
\hline
\end{tabular}

\subsection{P3 component}

Descriptive statistics for the P3 component are presented in Tables 2 and 3. Compared with the results obtained following $36 \mathrm{~h}$ of TSD, 8 h of RS was associated with significant increases in $\mathrm{P} 3$ amplitude $\left(F_{(1,13)}=8.674, P=0.011\right.$, 
$\left.\eta^{2}{ }_{P}=0.400\right)$, as well as significant decreases in P3 latency $\left(F_{(1 \otimes 13)}=14.778, P=0.002, \eta^{2}{ }_{P}=0.532\right)($ Fig. 3, Fig. 4C, D). P3 latency was most pronounced in the left and frontal electrodes, as indicated by the significant main effects of site $\left(F_{(2 \varangle 26)}=4.786, P=0.017, \eta^{2}{ }_{\mathrm{P}}=0.268\right)$ and region $\left(F_{(2 \varangle 26)}=28.625, P<0.001, \eta_{\mathrm{P}}^{2}=0.688\right)$. We also observed significant main effects of site on P3 amplitude, which was more positive in middle electrodes than in left electrodes $\left(F_{(2 \varangle 26)}=9.597, P=0.001, \eta^{2}{ }_{P}=0.425\right)$ (Fig. 3). No other main effects or interaction effects reached statistical significance.

Table 3

Grand-average peak amplitude of the N2 and P3 components for correct responses across multiple electrode sites after $36 \mathrm{~h}$ of TSD and after $8 \mathrm{~h}$ of RS

\begin{tabular}{|c|c|c|c|c|c|c|c|}
\hline & & $\mathrm{Oh}$ & & $36 \mathrm{~h}-\mathrm{T}$ & & $8 \mathrm{~h}-\mathrm{RS}$ & \\
\hline & & N2 & P3 & N2 & P3 & N2 & P3 \\
\hline F3 & $M(S D)$ & -3.61 & 7.94 & -3.50 & 6.23 & -3.40 & 9.23 \\
\hline & & $(4.23)$ & $(3.87)$ & (5.70) & $(4.40)$ & (3.09) & $(4.29)$ \\
\hline $\mathrm{Fz}$ & $M(S D)$ & -4.14 & 8.30 & -3.99 & 6.58 & -4.43 & 10.39 \\
\hline & & (4.63) & $(4.20)$ & (6.81) & (5.25) & (3.76) & (5.01) \\
\hline F4 & $M(S D)$ & -2.82 & 8.85 & -2.96 & 6.59 & -3.29 & 10.87 \\
\hline & & $(4.29)$ & $(4.25)$ & $(5.24)$ & $(5.09)$ & (3.76) & $(4.64)$ \\
\hline C3 & $M(S D)$ & -2.37 & 8.31 & -1.67 & 6.29 & -2.01 & 9.90 \\
\hline & & $(4.16)$ & (3.09) & (5.44) & (3.39) & $(2.54)$ & $(4.56)$ \\
\hline $\mathrm{Cz}$ & $M(S D)$ & -1.89 & 9.65 & -1.34 & 7.65 & -1.81 & 11.90 \\
\hline & & $(5.00)$ & (3.78) & $(6.23)$ & $(4.60)$ & (3.83) & $(4.88)$ \\
\hline C4 & $M(S D)$ & -0.45 & 9.87 & -0.35 & 7.96 & -0.63 & 11.66 \\
\hline & & (3.70) & $(3.35)$ & (4.77) & (3.80) & (3.14) & $(4.32)$ \\
\hline P3 & $M(S D)$ & -2.12 & 8.51 & -1.39 & 8.21 & -2.61 & 8.86 \\
\hline & & (6.17) & $(3.82)$ & (5.04) & (3.94) & (5.23) & (3.34) \\
\hline $\mathrm{Pz}$ & $M(S D)$ & -0.82 & 9.24 & 0.02 & 8.93 & -0.29 & 10.21 \\
\hline & & (4.13) & $(3.59)$ & $(4.43)$ & $(4.20)$ & $(4.18)$ & (3.25) \\
\hline P4 & $M(S D)$ & -0.36 & 7.93 & 0.32 & 8.47 & -0.93 & 8.38 \\
\hline & & (4.16) & $(3.41)$ & $(5.04)$ & (3.74) & $(4.30)$ & (3.14) \\
\hline
\end{tabular}

\section{Discussion}

In the present study, we analyzed ERPs to investigate the effects of $8 \mathrm{~h}$ of RS on working memory impairments induced by $36 \mathrm{~h}$ of TSD. Our previous results revealed that TSD significantly impairs accuracy in 2-back working memory tasks [43]. The current behavioral findings demonstrate that $8 \mathrm{~h}$ of RS can improve both accuracy and the number of correct responses per unit time in such tasks. Changes in behavioral indicators intuitively reflect 
increases in working memory ability. Although a constant cognitive load (2-back) was utilized in the present study, our results indicated that working memory capacity had improved following $8 \mathrm{~h}$ of RS, when compared with the capacity observed following $36 \mathrm{~h}$ of TSD without RS. However, emphasizing accuracy rates can also influence response times [47]. Although no improvements in reaction time were observed following $8 \mathrm{~h}$ of RS, the number of correct responses per unit time significantly increased. Therefore, we believe that the number of correct responses per unit of time more accurately reflects the individual's working memory ability and level of cognitive control.

Notably, 8 h of RS following TSD also induced significant decreases in N2/P3 latency, as well as significant increases in P3 amplitude. The N2 component is considered to reflect an individual's mental state and level of attention [18], while the P3 component is thought to be involved in the decision-making process during cognitivematching tasks [48]. Increases in P3 latency and decreases in P3 amplitude have been associated with prolonged wakefulness [49]. Numerous studies have reported that reaction time and sustained attention decrease following SD $[50,51]$. Thus, in accordance with previous results, our findings support the notion that $8 \mathrm{~h}$ of RS can improve performance and alertness [52]. Restoration of attention and alertness following RS may have enabled participants to allocate more attentional resources to the working memory tasks, thereby attenuating TSD-induced impairments [42]. Previous studies have also indicated that, relative to drowsiness, SD is associated with more pronounced decreases in activation of the frontoparietal network involved in working memory [53]. Furthermore, SD can reduce metabolic activity in regions associated with information processing and executive control [54], while RS can restore overall network organization following TSD [28].

Sustained attention and alertness are essential for performing daily activities. Based on the observed changes in the N2 and P3 components (i.e., increased amplitude and decreased latency) after $8 \mathrm{~h}$ of RS, we speculate that RS can effectively attenuate impairments in attention and alertness, thus influencing the information integration process. The deterioration of sustained attention seems to be the most long-lasting negative effect of SD [55, 56,] likely due to a decrease in general central nervous system (CNS) arousal $[57,58]$. In contrast, more automatic or bottom-up processes appear to be less affected by changes in CNS arousal [57]. Therefore, improvements in sustained attention are likely to occur earlier given the greater sensitivity of sustained attention to SD. The completion of a cognitive task usually requires the joint participation of several psychological processes, including early sensory perception, alertness, basic attentional mechanisms, working memory, and decision-making. The P3 component appears relatively late, suggesting that it is more reflective of conscious participation, which likely involves top-down cognitive control [59]. The observed increases in amplitude and decreases in latency also suggest that $8 \mathrm{~h}$ of RS improves the ability to integrate dynamic information during working memory tasks. Communication between the hippocampus and prefrontal areas is vital for optimal redistribution of temporal memory traces to more resident cortical storage, and interrupting this communication may impair the ability to form a new memory. However, Chai et al. recently noted that RS re-normalizes these hippocampal connections [34].

Normal sleep is divided into two phases: rapid eye movement (REM) and slow-wave sleep (SWS). Deep sleep during the N3 stage of SWS is particularly important for restoring mental and physical energy. Following SD, compensatory responses are observed during the restorative stages of sleep. Interestingly, it is the intensity rather than the duration of sleep that influences the recovery of function following SD. Sleep intensity during SWS is regarded as an indicator of homeostatic sleep stress $[29,60]$. After one night of SD, less than $10 \mathrm{~h}$ of RS can sufficiently decrease the level of sleep stress to that observed at the end of a typical 8-h period of normal sleep [61, 62]. Nevertheless, further increases in the duration of SWS are observed on the second night of recovery [63]. RS exhibits characteristics distinct from those of normal sleep, including decreases in sleep-onset latency. Key changes are also observed during the N2 and N3 stages. Longer periods of RS result in a more similar proportion of time spent in

Page $8 / 17$ 
sleep stages between normal sleep and RS [29]. Therefore, we speculate that individuals experienced increases in the proportion of SWS during RS, relative to the amount observed during normal sleep. It may be that SD-induced impairments in working memory function are specifically attenuated during SWS.

Although the present behavioral and EEG data support our hypothesis that $8 \mathrm{~h}$ of RS can attenuate impairments in working memory caused by $36 \mathrm{~h}$ of TSD, we did not observe significant changes in all indicators identified in our previous study [43]. Insufficient sleep may lead individuals to provide conservative estimates of their performance, which may increase the likelihood of compensatory behaviors and protect against the negative consequences of SD [33]. Therefore, the results of our study should be interpreted with caution. Studies have further demonstrated that simple cognitive responses are less affected by SD and can be easily recovered following RS, while impairments in higher-level cognitive functions are less easily reversed $[64,65]$. Improvements in cognitive function following RS are mainly reflected by changes in alertness and sustained attention, allowing participants to allocate more attentional resources to the current task [27]. Nonetheless, further studies are required to elucidate the mechanisms by which RS restores cognitive function following TSD.

The present study possesses some limitations of note. First, we did not assess working memory performance using tasks of varying difficulty, limiting our ability to infer how changes in workload impact the restorative effect of RS. In addition, our study included male volunteers only, necessitating caution when attempting to extend the findings to female individuals. Considering the small number of participants in our sample and some non-significant findings related to EEG indicators, further studies are required to determine whether $8 \mathrm{~h}$ of RS can restore cognitive function to baseline levels. In our future studies, we plan to include a control group to improve the rigor of our experimental design. Multimodal studies involving brain network analyses of EEG and imaging data may help to further explain our results. We did not utilize sleep monitoring technology to investigate whether RS induces specific alterations in sleep structure, necessitating further studies. Finally, given that circadian biorhythms affect behavioral performance [66], their effects cannot be excluded.

Our results align with those of previous studies, suggesting that $8 \mathrm{~h}$ of RS can partially attenuate the deleterious effects of TSD on working memory. Failure to maintain a level of alertness during military missions may lead to serious consequences. Under the conditions of future high-tech warfare, the problem of sleep deprivation may become more prominent. The widespread use of high-tech equipment puts forward higher requirements on the cognitive ability of the brain, therefore, it is of great military significance to attach importance to and strengthen the research on sleep deprivation and medical support under continuous combat conditions.

\section{Conclusion}

RS may exert its effects by improving alertness and sustained attention. Given that SWS dominates the sleep period during RS, these restored effects are likely to occur during these stages. However, as the effects of RS were limited in our study, further studies are required to determine whether $8 \mathrm{~h}$ of RS can restore cognitive function to baseline levels.

\section{List Of Abbreviations}

SD

sleep deprivation; ERPs:event-related potentials; TSD:total sleep deprivation; EEG:Electroencephalography; FPC:frontoparietal cortex; RON:the reorienting negativity; aMCC:anterior midcingulate cortex; PSQI:Pittsburgh Sleep 
Quality Index; ANOVA:analyses of variance; CNS:central nervous system; REM:rapid eye movement; SWS:slow-wave sleep.

\section{Declarations}

\section{Ethics approval and consent to participate}

This study was carried out in accordance with the recommendations of The Fourth Military Medical University with written informed consent from all subjects. The protocol was approved by The Fourth Military Medical University.

\section{Consent for publication}

Not applicable

\section{Availability of data and materials}

The dataset used in this study analysis is not currently available.

\section{Competing interests}

The authors declare they have no competing interests.

\section{Funding}

This research was supported by the National Science Foundation of Winter Olympics Technology Plan of China [grant number 2019YFF0301600].

\section{Author's Contributions}

YCS designed the study. ZYP produced the results and wrote the manuscript. Contributed data collection/analysis: YW, CMD, SYX, JLL, LTW and LJZ. YCS and WWF were the guarantors of this study. All authors listed have approved it for publication.

\section{Acknowledgements}

Not applicable

\section{References}

1. Anderson C, Horne JA. Sleepiness enhances distraction during a monotonous task. Sleep. 2006;29(4):573-6. doi: 10.1093/sleep/29.4.573. PMID: 16676792.

2. Djonlagic I, Rosenfeld A, Shohamy D, Myers C, Gluck M, Stickgold R. Sleep enhances category learning. Learn Mem. 2009;16(12):751-5. doi:10.1101/Im.1634509. PMID: 19926780; PMCID: PMC2788212.

3. Stickgold R. Sleep-dependent memory consolidation. Nature. 2005;437(7063):1272-8. doi: 10.1038/nature04286. PMID: 16251952.

4. Walker MP, Stickgold R. Sleep-dependent learning and memory consolidation. Neuron. 2004;44(1):121 - 33 . doi: 10.1016/j.neuron.2004.08.031. PMID: 15450165. 
5. Drummond SP, Paulus MP, Tapert SF. Effects of two nights sleep deprivation and two nights recovery sleep on response inhibition. J Sleep Res. 2006;15(3):261-5. doi: 10.1111/j.1365-2869.2006.00535.x. PMID: 16911028.

6. Alhola P, Polo-Kantola P. Sleep deprivation: Impact on cognitive performance. Neuropsychiatr Dis Treat. 2007;3(5):553-67. PMID: 19300585; PMCID: PMC2656292.

7. Anderson C, Platten CR. Sleep deprivation lowers inhibition and enhances impulsivity to negative stimuli. Behav Brain Res. 2011;217(2):463-6. doi:10.1016/j.bbr.2010.09.020. Epub 2010 Oct 1. PMID: 20888369.

8. Killgore WD, Balkin TJ, Wesensten NJ. Impaired decision making following $49 \mathrm{~h}$ of sleep deprivation. J Sleep Res. 2006;15(1):7-13. doi: 10.1111/j.1365-2869.2006.00487.x. PMID: 16489997.

9. Honn KA, Halverson T, Jackson ML, Krusmark M, Chavali VP, Gunzelmann G, et al. New insights into the cognitive effects of sleep deprivation by decomposition of a cognitive throughput task. Sleep. 2020;43(7):zsz319. doi:10.1093/sleep/zsz319. PMID: 32227081; PMCID: PMC7355397.

10. Smallwood J, Brown K, Baird B, Schooler JW. Cooperation between the default mode network and the frontalparietal network in the production of an internal train of thought. Brain Res. 2012;1428:60-70. doi: 10.1016/j.brainres.2011.03.072. Epub 2011 Apr 3. PMID: 21466793.

11. Wen X, Liu Y, Yao L, Ding M. Top-down regulation of default mode activity in spatial visual attention. J Neurosci. 2013;33(15):6444-53. doi:10.1523/JNEUROSCI.4939-12.2013. PMID: 23575842; PMCID: PMC3670184.

12. Baddeley A. Working memory. Curr Biol. 2010;20(4):R136-40. doi: 10.1016/j.cub.2009.12.014. PMID: 20178752.

13. Baddeley A. The episodic buffer: a new component of working memory? Trends Cogn Sci. 2000;4(11):417-423. doi: 10.1016/s1364-6613(00)01538-2. PMID: 11058819.

14. Gerhardsson A, Åkerstedt T, Axelsson J, Fischer H, Lekander M, Schwarz J. Effect of sleep deprivation on emotional working memory. J Sleep Res. 2019;28(1):e12744. doi:10.1111/jsr.12744. Epub 2018 Aug 8. PMID: 30091275; PMCID: PMC7379257.

15. Lo JC, Groeger JA, Santhi N, Arbon EL, Lazar AS, Hasan S, et al. Effects of partial and acute total sleep deprivation on performance across cognitive domains, individuals and circadian phase. PLoS One. 2012;7(9):e45987. doi:10.1371/journal.pone.0045987. Epub 2012 Sep 24. PMID: 23029352; PMCID: PMC3454374.

16. Zhang L, Shao Y, Liu Z, Li C, Chen Y, Zhou Q. Decreased Information Replacement of Working Memory After Sleep Deprivation: Evidence From an Event-Related Potential Study. Front Neurosci. 2019;13:408. doi:10.3389/fnins.2019.00408. PMID: 31105518; PMCID: PMC6499035.

17. Koslowsky M, Babkoff H. Meta-analysis of the relationship between total sleep deprivation and performance. Chronobiol Int. 1992;9(2):132-6. doi: 10.3109/07420529209064524. PMID: 1533178.

18. Schacht A, Werheid K, Sommer W. The appraisal of facial beauty is rapid but not mandatory. Cogn Affect Behav Neurosci. 2008;8(2):132 - 42. doi: 10.3758/cabn.8.2.132. PMID: 18589504.

19. Rosenthal L, Roehrs T, Zwyghuizen-Doorenbos A, Plath D, Roth T. Alerting effects of caffeine after normal and restricted sleep. Neuropsychopharmacology. 1991;4(2):103-8. PMID: 2025377.

20. Rogers PJ, Heatherley SV, Hayward RC, Seers HE, Hill J, Kane M. Effects of caffeine and caffeine withdrawal on mood and cognitive performance degraded by sleep restriction. Psychopharmacology (Berl). 2005;179(4):742 52. doi: 10.1007/s00213-004-2097-y. Epub 2005 Jan 26. PMID: 15887055.

21. Biggs SN, Smith A, Dorrian J, Reid K, Dawson D, van den Heuvel C, et al. Perception of simulated driving performance after sleep restriction and caffeine. J Psychosom Res. 2007;63(6):573-7.

doi:10.1016/j.jpsychores.2007.06.017. Epub 2007 Aug 1. PMID: 18061746.

Page 11/17 
22. Doty TJ, So CJ, Bergman EM, Trach SK, Ratcliffe RH, Yarnell AM, et al. Limited Efficacy of Caffeine and Recovery Costs During and Following 5 Days of Chronic Sleep Restriction. Sleep. 2017;40(12). doi: 10.1093/sleep/zsx171. PMID: 29029309.

23. Roehrs TA, Harris E, Randall S, Roth T. Pain sensitivity and recovery from mild chronic sleep loss. Sleep. 2012;35(12):1667-72. doi:10.5665/sleep.2240. PMID: 23204609; PMCID: PMC3490359.

24. Stroemel-Scheder C, Kundermann B, Lautenbacher S. The effects of recovery sleep on pain perception: A systematic review. Neurosci Biobehav Rev. 2020;113:408-25. doi:10.1016/j.neubiorev.2020.03.028. Epub 2020 Apr 8. PMID: 32275917.

25. Ruggiero JS, Redeker NS. Effects of napping on sleepiness and sleep-related performance deficits in night-shift workers: a systematic review. Biol Res Nurs. 2014;16(2):134 - 42. doi: 10.1177/1099800413476571. Epub 2013 Feb 13. PMID: 23411360; PMCID: PMC4079545.

26. Studte S, Bridger E, Mecklinger A. Nap sleep preserves associative but not item memory performance. Neurobiol Learn Mem. 2015;120:84-93. doi:10.1016/j.nIm.2015.02.012. Epub 2015 Feb 27. PMID: 25732251.

27. Jin X, Ye E, Qi J, Wang L, Lei Y, Chen P, et al. Recovery Sleep Reverses Impaired Response Inhibition due to Sleep Restriction: Evidence from a Visual Event Related Potentials Study. PLoS One. 2015;10(12):e0142361. doi:10.1371/journal.pone.0142361. PMID: 26658074; PMCID: PMC4684334.

28. Jiang Y, Chai Y, Yang F, Xu S, Basner M, Detre JA, et al. 0218 Effects Of Sleep Deprivation And Recovery Sleep On Human Brain Network Organization. Sleep (New York, N.Y.). 2018;41(suppl_1):A85-6. doi:10.1093/sleep/zsy061.217.

29. Hennecke E, Elmenhorst D, Mendolia F, Putzke M, Bauer A, Aeschbach D, et al. Reestablishment of individual sleep structure during a single 14-h recovery sleep episode after $58 \mathrm{~h}$ of wakefulness. J Sleep Res. 2019;28(3):e12641. doi:10.1111/jsr.12641.

30. Saletin JM, Goldstein-Piekarski AN, Greer SM, Stark S, Stark CE, Walker MP. Human Hippocampal Structure: A Novel Biomarker Predicting Mnemonic Vulnerability to, and Recovery from, Sleep Deprivation. J Neurosci. 2016 Feb 24;36(8):2355-63. doi: 10.1523/JNEUROSCI.3466-15.2016.

31. Banks S, Van Dongen HP, Maislin G, Dinges DF. Neurobehavioral dynamics following chronic sleep restriction: dose-response effects of one night for recovery. Sleep. 2010;33(8):1013-26. doi:10.1093/sleep/33.8.1013.

32. Lo JC, Ong JL, Leong RL, Gooley JJ, Chee MW. Cognitive Performance, Sleepiness, and Mood in Partially Sleep Deprived Adolescents: The Need for Sleep Study. Sleep. 2016;39(3):687-98. doi:10.5665/sleep.5552.

33. Boardman JM, Bei B, Mellor A, Anderson C, Sletten TL, Drummond SPA. The ability to self-monitor cognitive performance during $60 \mathrm{~h}$ total sleep deprivation and following 2 nights recovery sleep. J Sleep Res. 2018;27(4):e12633. doi:10.1111/jsr.12633.

34. Chai Y, Fang Z, Yang FN, Xu S, Deng Y, Raine A, et al. Two nights of recovery sleep restores hippocampal connectivity but not episodic memory after total sleep deprivation. Sci Rep. 2020;10(1):8774. doi:10.1038/s41598-020-65086-x.

35. Gumenyuk V, Howard R, Roth T, Korzyukov O, Drake CL. Sleep loss, circadian mismatch, and abnormalities in reorienting of attention in night workers with shift work disorder. Sleep. 2014;37(3):545-56. doi:10.5665/sleep.3494.

36. Folstein JR, Van Petten C. Influence of cognitive control and mismatch on the N2 component of the ERP: a review. Psychophysiology. 2008;45(1):152-70. doi:10.1111/j.1469-8986.2007.00602.x. 
37. Huster RJ, Westerhausen R, Pantev C, Konrad C. The role of the cingulate cortex as neural generator of the N200 and P300 in a tactile response inhibition task. Hum Brain Mapp. 2010;31(8):1260-71. doi:10.1002/hbm.20933.

38. Verleger R, Jaśkowski P, Wascher E. Evidence for an Integrative Role of P3b in Linking Reaction to Perception. Journal of psychophysiology. 2005;19(3):165-81.

39. Polich J. Updating P300: an integrative theory of P3a and P3b. Clin Neurophysiol. 2007;118(10):2128-48. doi:10.1016/j.clinph.2007.04.019.

40. Raud L, Huster RJ. The Temporal Dynamics of Response Inhibition and their Modulation by Cognitive Control. Brain Topogr. 2017;30(4):486-501. doi:10.1007/s10548-017-0566-y.

41. Dehaene S, Changeux JP. Experimental and theoretical approaches to conscious processing. Neuron. 2011;70(2):200-27. doi:10.1016/j.neuron.2011.03.018.

42. Donchin E, Fabiani M. The use of event-related brain potentials in the study of memory: Is P300 a measure of event distinctiveness?. C Massachusetts Institute of Technology. Center for International Studies. 1991;44: 5877.

43. Peng Z, Dai C, Ba Y, Zhang L, Shao Y, Tian J. Effect of Sleep Deprivation on the Working Memory-Related N2-P3 Components of the Event-Related Potential Waveform. Front Neurosci. 2020;14:469. doi:10.3389/fnins.2020.00469.

44. Buysse DJ, Reynolds CF 3rd, Monk TH, Berman SR, Kupfer DJ. The Pittsburgh Sleep Quality Index: a new instrument for psychiatric practice and research. Psychiatry Res. 1989;28(2):193-213. doi:10.1016/01651781(89)90047-4.

45. Dinges DF. Are you awake? Cognitive performance and reverie during the hypnopompic state. Sleep and cognition Washington, DC: American Psychological Association 1990;159 - 75.

46. Dinges DF. Differential effects of prior wakefulness and circadian phase on nap sleep. Electroencephalogr Clin Neurophysiol. 1986;64(3):224-7. doi:10.1016/0013-4694(86)90170-7.

47. de Bruijn ERA, Jansen M, Overgaauw S. Enhanced error-related brain activations for mistakes that harm others: ERP evidence from a novel social performance-monitoring paradigm. Neuroimage. 2020;204:116238. doi:10.1016/j.neuroimage.2019.116238.

48. Gosselin A, De Koninck J, Campbell KB. Total sleep deprivation and novelty processing: implications for frontal lobe functioning. Clin Neurophysiol. 2005;116(1):211-22. doi:10.1016/j.clinph.2004.07.033.

49. Panjwani U, Ray K, Chatterjee A, Bhaumik S, Kumar S. Electrophysiological correlates of cognition improve with nap during sleep deprivation. Eur J Appl Physiol. 2010;108(3):549-56. doi:10.1007/s00421-009-1222-3.

50. Rupp TL, Wesensten NJ, Bliese PD, Balkin TJ. Banking sleep: realization of benefits during subsequent sleep restriction and recovery. Sleep. 2009;32(3):311-21. doi:10.1093/sleep/32.3.311.

51. Chua EC, Yeo SC, Lee IT, Tan LC, Lau P, Cai S, et al. Sustained attention performance during sleep deprivation associates with instability in behavior and physiologic measures at baseline. Sleep. 2014;37(1):27-39. doi:10.5665/sleep.3302.

52. Zhang Q, Liao Y, Qi J, Zhao Y, Zhu T, Liu Z, et al. A visual ERP study of impulse inhibition following a zaleploninduced nap after sleep deprivation. PLoS One. 2014;9(5):e95653. doi:10.1371/journal.pone.0095653.

53. Almklov EL, Drummond SP, Orff H, Alhassoon OM. The effects of sleep deprivation on brain functioning in older adults. Behav Sleep Med. 2015;13(4):324-45. doi:10.1080/15402002.2014.905474.

54. Choo WC, Lee WW, Venkatraman V, Sheu FS, Chee MW. Dissociation of cortical regions modulated by both working memory load and sleep deprivation and by sleep deprivation alone. Neuroimage. 2005;25(2):579-87. 
doi:10.1016/j.neuroimage.2004.11.029.

55. de Bruin EJ, van Run C, Staaks J, Meijer AM. Effects of sleep manipulation on cognitive functioning of adolescents: A systematic review. Sleep Med Rev. 2017;32:45-57. doi:10.1016/j.smrv.2016.02.006.

56. Lowe CJ, Safati A, Hall PA. The neurocognitive consequences of sleep restriction: A meta-analytic review. Neurosci Biobehav Rev. 2017;80:586-604. doi:10.1016/j.neubiorev.2017.07.010.

57. Schneider W, Fisk AD. Automatic category search and its transfer. J Exp Psychol Learn Mem Cogn. 1984;10(1):1-15. doi:10.1037//0278-7393.10.1.1.

58. Cote KA, Milner CE, Smith BA, Aubin AJ, Greason TA, Cuthbert BP, et al. CNS arousal and neurobehavioral performance in a short-term sleep restriction paradigm. J Sleep Res. 2009;18(3):291-303. doi:10.1111/j.13652869.2008.00733.x.

59. Kusztor A, Raud L, Juel BE, Nilsen AS, Storm JF, Huster RJ. Sleep deprivation differentially affects subcomponents of cognitive control. Sleep. 2019;42(4):zsz016. doi:10.1093/sleep/zsz016.

60. Borbély AA. A two process model of sleep regulation. Hum Neurobiol. 1982;1(3):195-204. PMID: 7185792.

61. Achermann P, Borbély AA. Simulation of daytime vigilance by the additive interaction of a homeostatic and a circadian process. Biol Cybern. 1994;71(2):115-21. doi:10.1007/BF00197314.

62. Daan S, Beersma DG, Borbély AA. Timing of human sleep: recovery process gated by a circadian pacemaker. Am J Physiol. 1984;246(2 Pt 2):R161-83. doi:10.1152/ajpregu.1984.246.2.R161.

63. Carskadon MA, Dement WC. Sleep loss in elderly volunteers. Sleep. 1985;8(3):207-21. doi:10.1093/sleep/8.3.207.

64. Nilsson JP, Söderström M, Karlsson AU, Lekander M, Akerstedt T, Lindroth NE, et al. Less effective executive functioning after one night's sleep deprivation. J Sleep Res. 2005;14(1):1-6. doi:10.1111/j.13652869.2005.00442.x.

65. Skurvydas A, Zlibinaite L, Solianik R, Brazaitis M, Valanciene D, Baranauskiene N,et al. One night of sleep deprivation impairs executive function but does not affect psychomotor or motor performance. Biol Sport. 2020;37(1):7-14. doi:10.5114/biolsport.2020.89936.

66. Montplaisir J. [Depression and biological rhythm: therapeutic effects of sleep deprivation]. Union Med Can. 1981;110(3):272-6. French. PMID: 7245424.

\section{Figures}




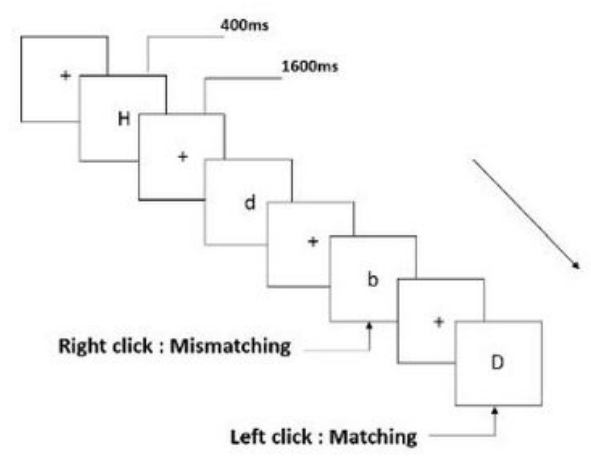

(a)

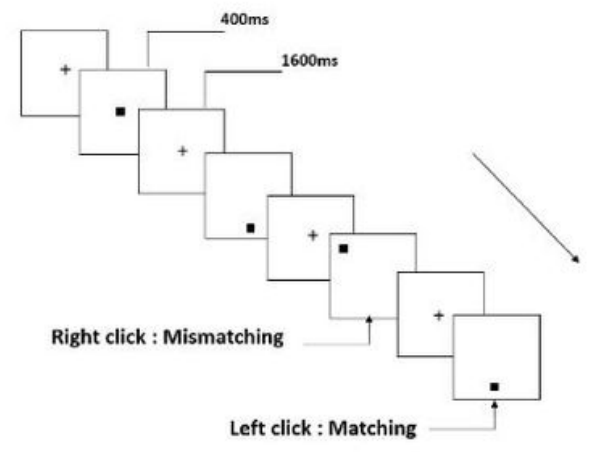

(b)
The three independent tasks are ordered left-right as follows:

(a)pronunciation working memory task, (b)spatial working memory task,

(c)object working memory task.

The three tasks are presented successively.

Participants are required to complete all types of tasks

at 3 time points (before TSD, after TSD, after RS)

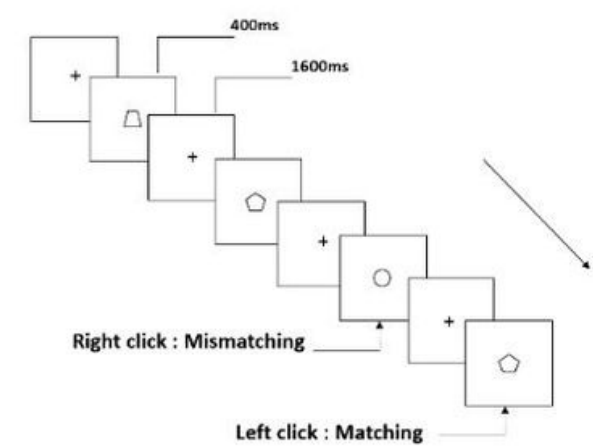

(c)

Figure 1

Schematic diagram of the working memory task

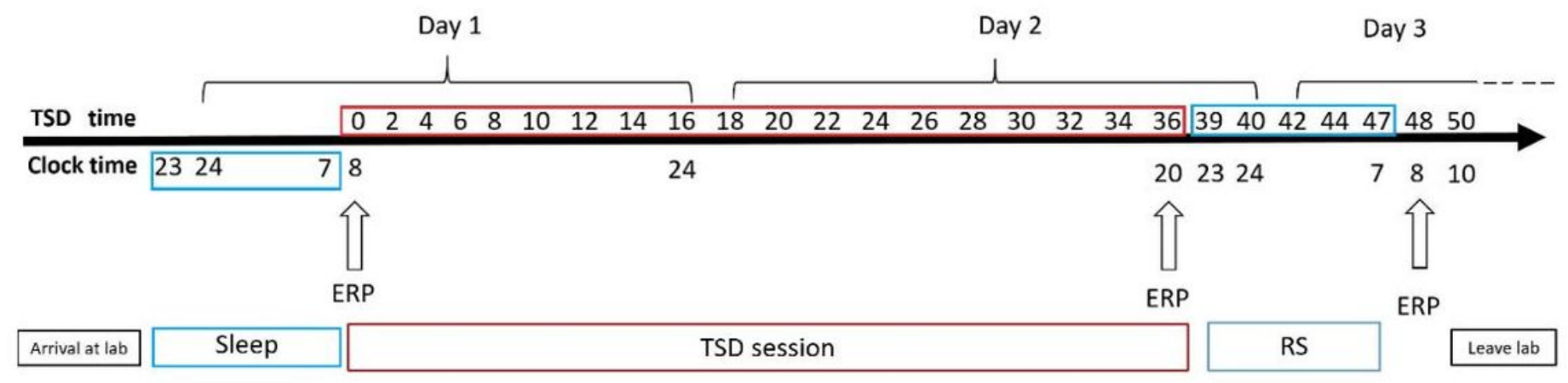

\section{Figure 2}

Experimental design. After $8 \mathrm{~h}$ of sleep in our laboratory, participants underwent $36 \mathrm{~h}$ of TSD, followed by $8 \mathrm{~h}$ of RS. EEG data were recorded simultaneously. The arrows indicate the time points during the 2-back working memory task. TSD: total sleep deprivation; RS: recovery sleep; EEG: electroencephalography. 

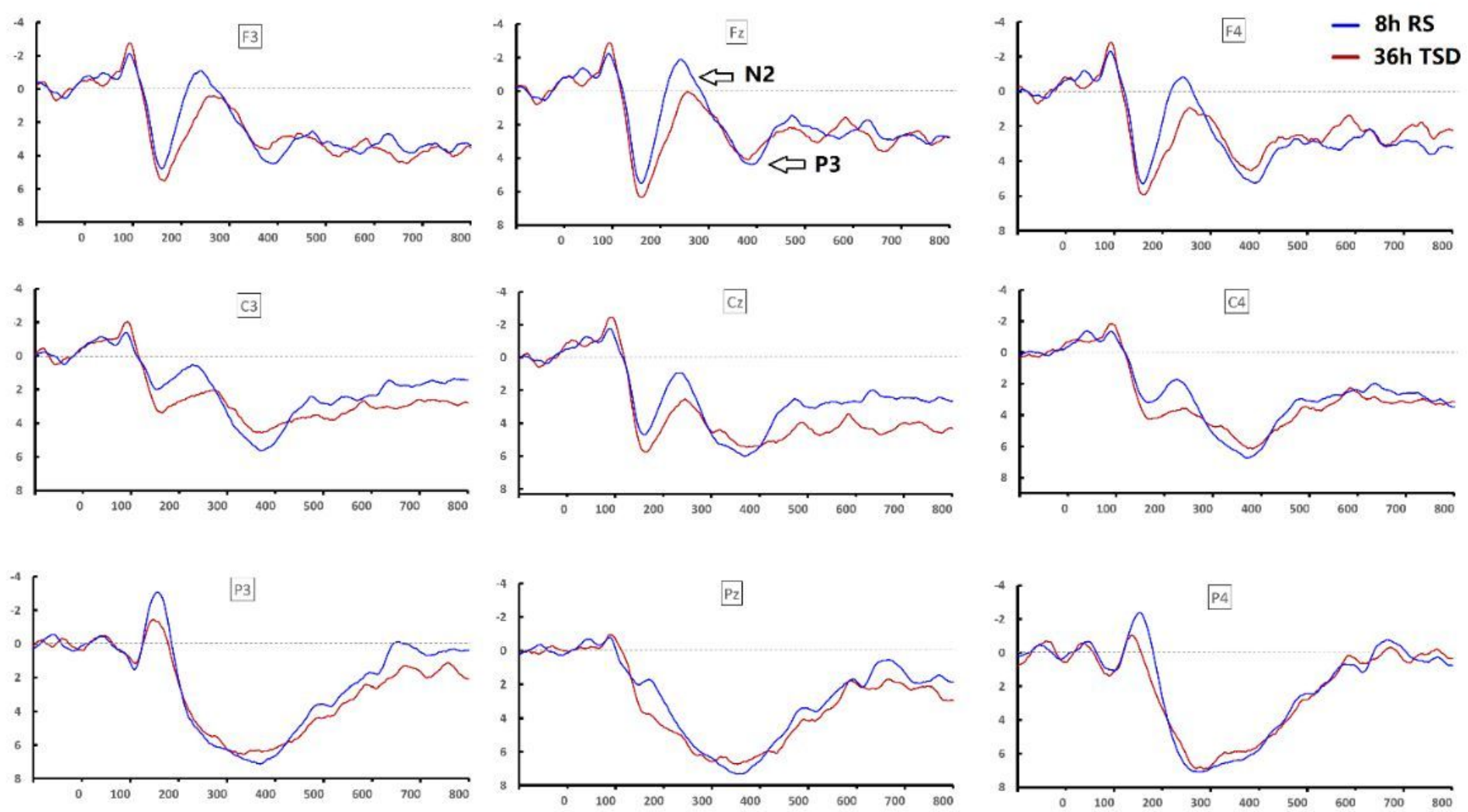

\section{Figure 3}

ERP amplitude for correct responses during the working memory tasks after $36 \mathrm{~h}$ of TSD and $8 \mathrm{~h}$ of RS. The channels are ordered from left to right and top to bottom as follows: F3, Fz, F4, C3, Cz, C4, P3, Pz, P4. ERP: eventrelated potential (ERP). The ERP waveform is obtained by superimposing all trials in which correct responses were observed (consistent and inconsistent) (RS: consistent: 35, inconsistent 32; TSD: consistent: 30, inconsistent 29). TSD: total sleep deprivation; RS: recovery sleep. 


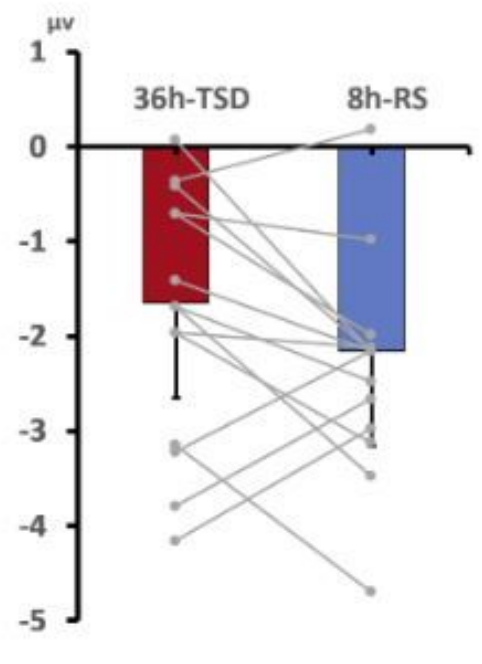

(A)N2 amplitude

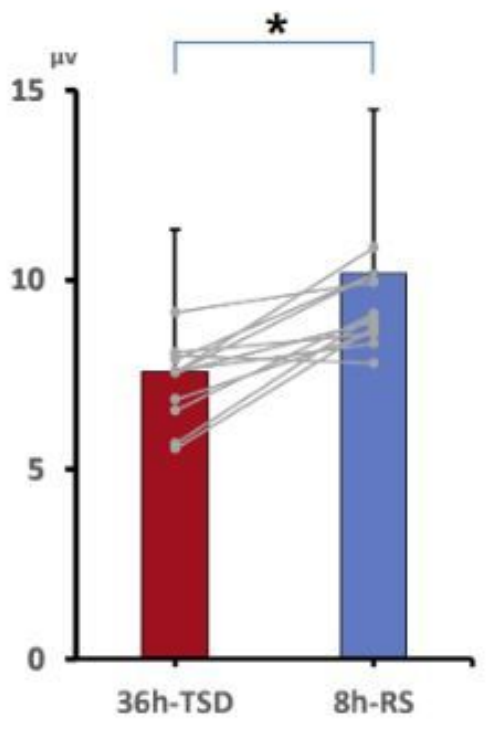

(C)P3 amplitude

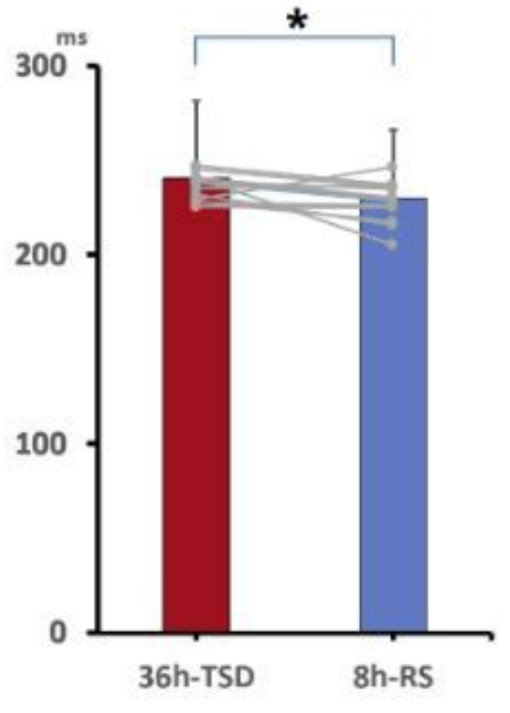

(B) N2 latency

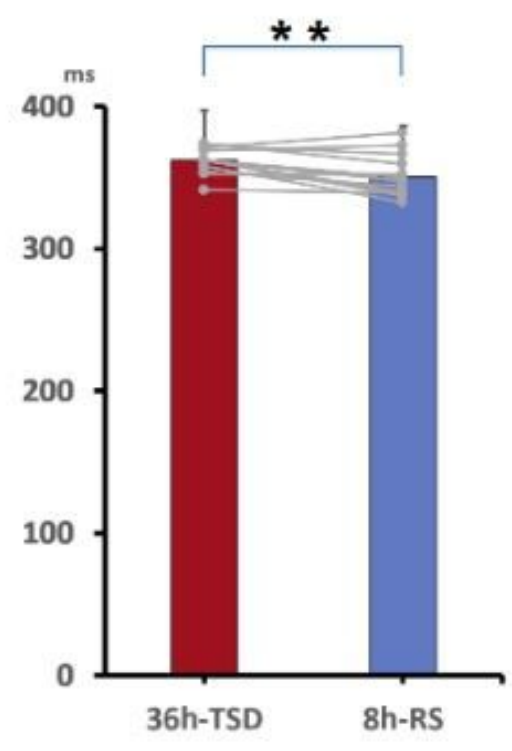

(D)P3 latency

\section{Figure 4}

Group-level (bar graph) and individual-level (circles) differences in ERP components between $36 \mathrm{~h}$ of TSD and $8 \mathrm{~h}$ of RS. (A) Mean N2 amplitude after $36 \mathrm{~h}$ of TSD and $8 \mathrm{~h}$ of RS. (B) Mean N2 latency after $36 \mathrm{~h}$ of TSD and $8 \mathrm{~h}$ of RS. (C) Mean P3 amplitude after $36 \mathrm{~h}$ of TSD and $8 \mathrm{~h}$ of RS. (D) Mean P3 latency after $36 \mathrm{~h}$ of TSD and $8 \mathrm{~h}$ of RS. Error bars represent the standard deviation. ${ }^{*} p<0.05,{ }^{* *} p<0.01$. ERP: event-related potential (ERP); TSD: total sleep deprivation; RS: recovery sleep. 36hTSD: after $36 \mathrm{~h}$ of TSD; 8hRS: after $8 \mathrm{~h}$ of RS. 